\title{
The Application of MAS towards Simulation of Forest Fire Spreading
}

\author{
Zuowei Huang ${ }^{1,2}$ and Fang Liu ${ }^{2}$ \\ ${ }^{1}$ School of Architecture and urban planning, Hunan University of Technology, \\ Zhuzhou 412008, China \\ ${ }^{2}$ School of Geosciences and Information-Physics, \\ Central South University, Changsha 410083, China \\ huangzuowei4@126.com
}

\begin{abstract}
With the rapid development of spatial information technology and the increasingly artificial intelligence knowledge, multi-Agent Systems technology plays a more and more important role in conducting fire behavior forecast, in order to simplify the complex mechanism of fire spreading and improve the simulation speed, according to the law of conservation of energy, fire spreading Agent model was set up. This paper, which analyzing comprehensively fire spreading model and some influenced factors, is concentrated on how to stimulate fire spreading regularity base on the knowledge of mathematics and computer, an interactive fire spreading simulation model based on MAS and GIS information feedback mechanism is put forward., Fire spreading Agents participating in it can exchange knowledge and information with each other, and achieve a consistent decision to form an acceptable scheme through feedbacks and coordination. Finally the results show that this model not only better simulates fire spreading under different scenarios, but also provides effectively solutions to fire suppression planning.
\end{abstract}

Keywords: Fire spreading, dynamic monitoring, MAS, simulation, GIS

\section{Introduction}

Fire spreading has the feature of time and space. With the rapid development of $3 \mathrm{~S}$ spatial information technology and the increasingly growth knowledge of fire disaster , It pays a more and more important role in conducting fire behavior forecast within the field of fire prevention by means of GIS technology. Fire behavior pattern is closely related with the change of fire intensity [1]. Owing to changeable atmosphere condition, sophisticated terrain and Vegetation distribution, even different fire spreading direction can cause the obviously change of fire intensity, so cause the change of fire pattern [2,4]. It is difficult to describe it in a general conception since the affected factor of fire behaviors is varied and its complex mechanism. By means of MAS and GIS object-oriented as a tool which studies the change law and spatial pattern of fire behavior is an important method for the future $[5,6,7]$.

Multi-agent is an important branch of distributed artificial intelligence. It is developed to solve the large-scale complex problems intelligently. Its basic idea is to separate large complex systems into many small autonomy systems (agents), which can communicate with each other and operate coordinately $[8,9]$. Through cooperation with the agent's intelligent behaviors, such as interaction and collaboration, complex task can be solved. The application of multi-agent technology in forest firing is still in the exploratory stage. Most researches are 
still in the concept proposed and model designed stages. Few of them can be applied to the actual structure. scholars have studied and developed a series of fire spreading simulation models, which can be divided into several groups, including the Equation-Based Models, System Models, Statistical Models, Evolution Models, Cellular Models, Agent-Based Models and so on (Liu, et al., 2008; Chen, 2010). These simulation methods have their own advantages and disadvantages. The equation-based model regarded as a kind of static model is easy for quantitative analysis, but does not consider spatial complexity simulation of fire spreading (Parker, et al., 2003)[10]. The model has strong ability of systematic analysis, but is difficult to achieve spatial analysis. The evolutionary model is a kind of effective method to solve quantitatively decision problems because of its simplicity, common use, strong robustness and the ability of parallel computing [11-13]. However, it is inclined to fall into locally optimal solution when the problem is more complex. Due to the agent itself has characteristics of initiative, interactive, collaborative, reactivity, autonomy, mobility, it is more suitable for expressing complicated group behavior, in particular, have great advantages over geographical spatio-temporal related events.

\section{Fire Spreading Model based on Multi-Agent Systems}

\subsection{The hierarchical structure of the model}

The model can be divided into five levels, as shown in Figure 1, can be expressed as follows :

FSDSS $=\langle$ DSSM, A, UI, G, LR >

Where DSSM represents the decision support system for comprehensive Agent management and task distribution.

A -collection of decision-making set, $\mathrm{A}=\{\mathrm{Ai}, \mathrm{i}=1,2, \ldots, \mathrm{n}\}$, each decision-making agent has its own functions and duties, work together to provide decision support for fire spreading.

UI-the user interface, which is used to receive the user's decision-making and process various manual and instructions in the system.

G-attribute of agent, Including decision agent, Management Agent, Knowledge Agent, Information Agent and Collaborative Agent and so on.

LR-local decision resources, that is, including data, models, knowledge, documentation, resource collections all can aid decision- making resources and tools, it is saved in the three local libraries (model Libraries, knowledge base and database) and used by decision makers.

Collaborative agent: collaborative agent including all kinds of cooperation agreements, policies, handling of the coordination and evaluation. Collaboration strategy refers to decisions and control process between the Agent, and aims to seek a method both sides can accept the strategy. Collaborative processing refers to the specific behavior during the process of collaboration. It make comprehensive coordination and to propose the fire spreading scheme of promoting agents to achieve a consistent decision. Cell selection results and the evaluation to other agents' cell selection results are required to communicate with collaborative agent.

Information Agent: retrieve and process all kinds of information, it can realize the management, control and classification of fire spreading information. Information Agent retrieve the information for policymakers according to the need from the information source, agents have such behaviors as observation, perception, comparison and evaluation, and can 
express the particular desires based on background and knowledge. These desires determine the observation content of agents in the system.

Management Agent: The functions of Management Agent are the decision of the question, conduct coordination with the fire spreading factors from the different group. It needs to solve the difficult problem such as task decomposition, task allocation, consultations between the Agent. based on user requirements Management Agent develop mission planning and consult with other subsystem, After the process of observation, perception, comparison and evaluation, it will select the interested fire unit as the preference locations, and these locations can also be selected by other agents in the model.

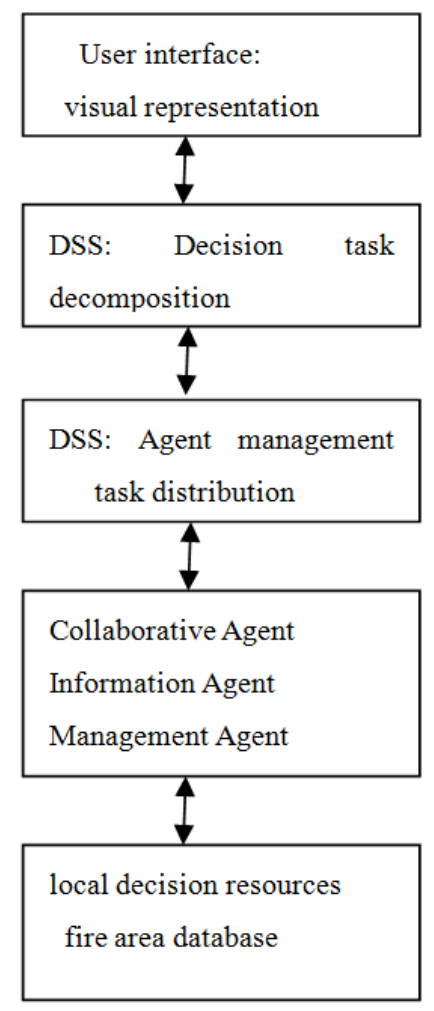

Figure 1. Hierarchical structure of the model

\subsection{Model overview}

Multi-Agent can be a physical entity, such as in the Cartesian task environment it operates objects work together cooperatively, which move them from one location to another location, intelligent body can also be calculation code, such as optimizing agents, they could work together to examine certain values and can effectively narrow the search space to achieve a series of small space. Regardless of the domain on agent, one thing is the same, namely, during the problem-solving processing; it conducts local physics or computing interactively in task space. In response to the different local constraints received from the task space, the agent can select and show different behavior patterns. Multi-agent is composed of multiple autonomous agents, which has problem -solving ability and interact with each other to achieve the overall goal. Due to the above characteristics of multi-agent body, so it can be applied to the Forest fire spreading simulation.

The model consists of Fire spreading Decision Support System and multi-agent systems, 
and both systems are interacted through unified cells (Figure 2). The agents are divided into Knowledge Agent, Information Agent and Collaborative Agent. Information agents have such behaviors as observation, perception, comparison and evaluation, and can express the particular desires based on background and knowledge. These desires determine the observation content of Information agents in the spatial system. The observation result is called a fact. By perceiving and evaluating facts, Information agent will form the perception directly related to the state of fire spreading system. Each Information agent will compare its own perception with perception of other Information agents and evaluate all perceptions of other Information agents relative to its own perception. Comparison and evaluation information will be communicated with Collaborative agent.

In order to guarantee the convergence of the model, improve the speed that agents achieve a consistent decision, and ensure that information agents can express the application requirements in time and receive feedback information, the model conforms to the following two assumed conditions.

(1) Each agent should follow a set of predefined desire rule, in which new desires can not be added and utility function cannot be dynamically changed.

(2) All agents in the model can equally and fully utilize all information of decision- making system.

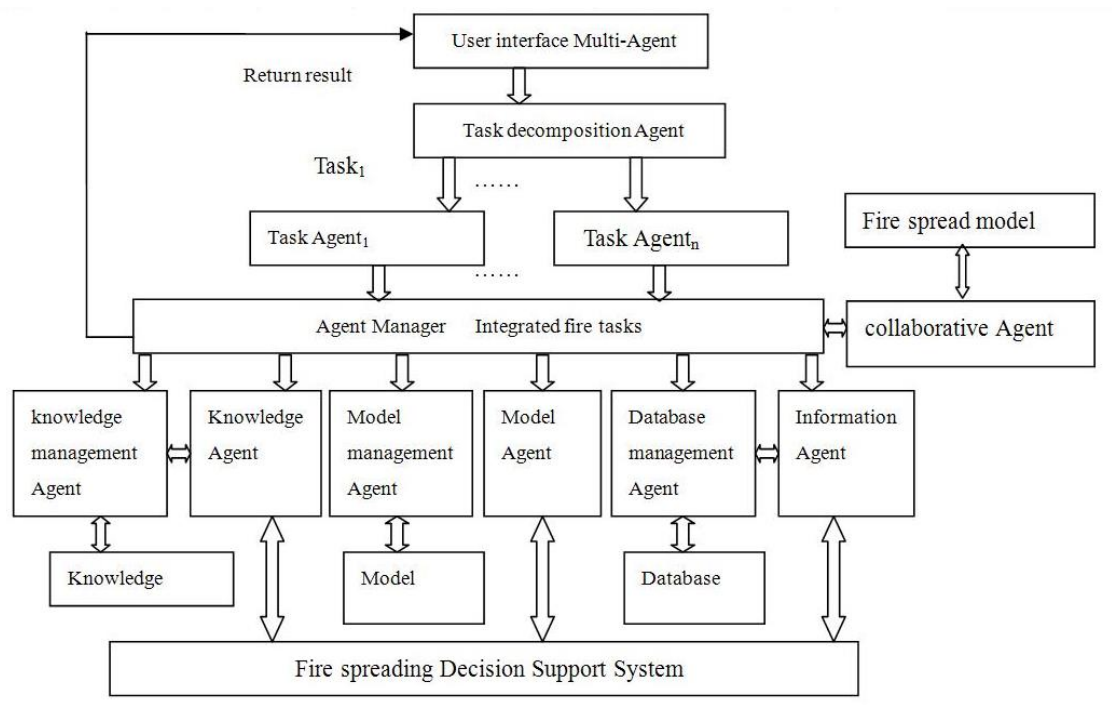

Figure 2. The General Framework of interactive fire spread model based on MAS

\section{The Building of Fire Spreading Model}

During the flammable forests area $U(x, y)$, it take the temperature field as the state of the fire, considering the effect of heat conduction, convection, radiation, and wind direction, the following equation derived from the law of conservation of energy :

Combustion gross heat quantity $\left(q_{\text {总 }}\right)=$ temperatures rise required energy $\left(q_{1}\right)+$ scattering around energy $\left(q_{2}\right)+$ outward radiation energy $\left(q_{3}\right)+$ air convection energy

$\left(q_{4}\right)+$ the heat taken away by the wind $\left(q_{5}\right)$. In the middle $q_{\text {总 }}$ represents the energy 
emitted by the burning area, per unit volume and per unit time the combustion heat quantity $Q$, within a certain time the burning of combustion energy $Q_{\text {总 }}=Q d x d y d z$, then micro-unit of calorific value is:

$$
q_{\text {总 }}=\int_{\tau} Q_{\text {总 }} d \tau
$$

$q_{1}$ represents the required energy temperatures rise in the combustion region, it can increase reaction in micro unit physical quantity storage over time, during the time $\Delta \tau$ in the combustion region after temperatures rise it set temperature $t_{e}$, and before it set temperature $t_{s}$, so the change temperature $\Delta t=t_{e}-t_{s}$, so the gas heating up required heat with time change rate is: $Q_{1}=\rho_{i} c_{i} \frac{\partial t}{\partial \tau} d x d y d z$

$$
q_{1}=\int_{\tau} Q_{1} d \tau
$$

where $\rho_{i}$ represents the density of the gas in the region; $c_{i}$ represents the heat capacity of the gas in the region.

$q_{2}$ represents dissipating heat into the surrounding space in micro-unit body, any high temperature object has the ability of heat transfer into the surrounding space, for every agent body $d x d y d z$, fire spreading Agent is shown in Figure 3.

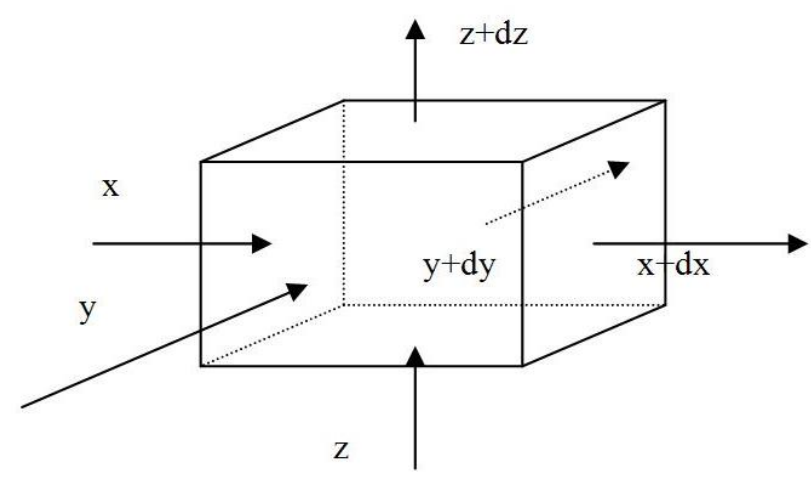

Figure 3. Agent of Fire Spreading

Any direction of heat flow can be decomposed into $x, y, z$ three axis of heat flow, and through $x=x, y=y, z=z$ the three surface into micro-unit body heat, according to the law of Fourier it can directly draw heat conduction differential equations as follows:

$$
\left\{\begin{array}{l}
Q_{x}=-\lambda \frac{\partial t}{\partial x} d y d z \\
Q_{y}=-\lambda \frac{\partial t}{\partial y} d x d z \\
Q_{z}=-\lambda \frac{\partial t}{\partial z} d x d y
\end{array}\right.
$$


Through $x=x+d x, y=y+d y, z=z+d z$, the same method it can draw following equation:

$$
\left\{\begin{array}{l}
Q_{x+d x}=-\lambda \frac{\partial}{\partial x}\left(t+\frac{\partial t}{\partial x} d x\right) d y d z \\
Q_{y+d y}=-\lambda \frac{\partial}{\partial y}\left(t+\frac{\partial t}{\partial y} d y\right) d x d z \\
Q_{z+d z}=-\lambda \frac{\partial}{\partial z}\left(t+\frac{\partial t}{\partial z} d z\right) d x d y
\end{array}\right.
$$

Because micro-unit derived the sum total of the heat outside $=$ export the total quantity heat of micro-unit - import total quantity heat of micro-unit, namely:

$$
\begin{gathered}
Q_{2}=Q_{x+d x}-Q_{x}+Q_{y+d y}-Q_{y}+Q_{z+d z}-Q_{z}=-\lambda\left(\frac{\partial^{2} t}{\partial x^{2}}+\frac{\partial^{2} t}{\partial y^{2}}+\frac{\partial^{2} t}{\partial z^{2}}\right) d x d y d z \\
q_{2}=\int_{\tau} Q_{2} d \tau
\end{gathered}
$$

$\mathrm{Q}_{3}$ represents radiation energy outward in micro body, the process of transfer of energy called radiant energy, in the process of combustible burning, burning flammable material can be regarded as an outward radiation energy of high temperature object. it can draw following equation:

$$
\begin{aligned}
Q_{3} & =Q_{0}+Q_{01} \\
& =x_{2,1} \varepsilon_{b} C_{0}\left[\left(T_{1} / 100\right)^{4}-\left(T_{2} / 100\right)^{4}\right] d s_{2}+\left(1-x_{1,2}\right) \varepsilon_{b} C_{0}\left(T_{1} / 100\right)^{4} \frac{x_{2,1}}{x_{1,2}} d s_{2} \\
& =\varepsilon_{b} C_{0}\left[\left(x_{1,2}+\frac{x_{2,1}}{x_{1,2}}-x_{2,1}\right)\left(T_{1} / 100\right)^{4}-x_{1,2}\left(T_{2} / 100\right)^{4}\right] d s_{2}
\end{aligned}
$$

Among them: $\mathrm{C}_{0}$ represents the blackbody radiation coefficient; $\varepsilon_{b}$ represents the orientation degrees of the black object; $x_{1,2}, x_{2.1}$ represents Angle coefficient; $T_{1}, T_{2}$ represents temperature.

$$
q_{3}=\int_{\tau} Q_{3} d \tau
$$

$\mathrm{q}_{4}$ represents the energy consumption for air convection. when the slope is zero, in the process of fuel burning, hot air rising, which formed the convection gas. Suppose at a certain time the burning zone temperature equal with the convection zone. the loss energy of the convection unit time is:

$$
\begin{aligned}
Q_{4}=\rho_{i} c_{i} u\left(t_{e 0}-t_{s}\right) d x d y & =\rho_{i} c_{i} u d t d x d y \\
q_{4} & =\int_{\tau} Q_{4} d t
\end{aligned}
$$


Wind affect the air flow, air flow causes the flow of heat, as a result, the flow of the wind will take away part of the heat, and the heat taken away by the wind unit time is:

$$
\begin{array}{r}
Q_{5}=\rho_{i} c_{i}\left(v_{x} d t d y d z+v_{y} d t d x d z\right) \\
q_{5}=\int_{\tau} Q_{5} d \tau
\end{array}
$$

Where $\rho_{i}$ represents density of air, ${ }^{c_{i}}$ represents specific heat capacity of air; $v_{x}$ represents the component of wind speed on the axes $\mathrm{x}$; $v_{y}$ represents the component of wind speed on the axis $y$.

On the base of the above analysis, According to the law of energy conservation it can be obtained the following equation:

$$
\int_{\tau} Q_{\text {总 }} d \tau=\int_{\tau} Q_{1} d \tau+\int_{\tau} Q_{2} d \tau+\int_{\tau} Q_{3} d \tau+\int_{\tau} Q_{4} d t+\int_{\tau} Q_{5} d \tau
$$

Through differential on both sides at the same time:

Because of fire spreading are extremely complex, its energy conversion is varied, in terms of its energy transmission, the above of burning area heat : $Q_{\text {上 }}=-\lambda \frac{\partial^{2} t}{\partial z^{2}}$, for each burning point $(x, y)$, during the combustion process, the heat in all directions account for the proportion of the total heat approximately remain the same, in the actual calculation, to simplify :

$$
k_{1}=\frac{Q_{\text {上 }}}{Q}
$$

Similarly, the above of burning area convection heat : $k_{2}=\frac{Q_{4}}{Q}$

In order to calculate the burning area of fire, set start burning point $(0,0)$, at time $\tau=0$ it begin burning. Defines $K^{+}$represents the set of combustion boundary point when $\mathrm{y}>0$.

$$
\begin{gathered}
K^{+}=\left\{(x, y, \tau) \mid(x, y, \tau) \in I, t(x, y, \tau)>t_{r},\left[t(x+\Delta x, y, \tau)<t_{r}\right.\right. \\
\left.\left.t(x, y+\Delta y, \tau)<t_{r}\right], x * \Delta x>0, \Delta y>0\right\}
\end{gathered}
$$

The set represents there are boundaries of $(\mathrm{x}, \mathrm{y})$ correspond to each time,

Similarly, the set of combustion boundary point when $\mathrm{y}<0$ :

$$
\begin{aligned}
K^{-}= & \left\{(x, y, \tau)(x, y, \tau) \in I, t(x, y, \tau)>t_{r},\left[t(x+\Delta x, y, \tau)<t_{r}\right.\right. \\
& \left.\left.t(x, y+\Delta y, \tau)<t_{r}\right], x * \Delta x>0, \Delta y<0\right\}
\end{aligned}
$$

Part area of $y>0$ :

$$
S_{\tau}^{+}=\int_{x_{l \tau}}^{x_{r \tau}} f_{\tau}(x) d x
$$


Part area of $y<0$ :

$$
S_{\tau}^{-}=\int_{x_{l \tau}}^{x_{r \tau}} \varphi_{\tau}(x) d x
$$

Then the total burning area:

$$
S_{\tau}=S_{\tau}^{+}+S_{\tau}^{-}=\int_{x_{l \tau}}^{x_{r \tau}} f_{\tau}(x) d x+\int_{x_{l \tau}}^{x_{r \tau}} \varphi_{\tau}(x) d x
$$

It assumed time $\tau_{1}$ and $\tau_{2} \quad\left(\tau_{2}>\tau_{1}\right)$,forest fire burning area as follows:

$$
\begin{aligned}
& S_{\tau 1}=\int_{x_{l \tau 1}}^{x_{r \tau 1}} f_{\tau 1}(x) d x+\int_{x_{l \tau 1}}^{x_{r \tau 1}} \varphi_{\tau 1}(x) d x \\
& S_{\tau 2}=\int_{x_{l \tau 2}}^{x_{r \tau 2}} f_{\tau 2}(x) d x+\int_{x_{l \tau 2}}^{x_{r \tau 2}} \varphi_{\tau 2}(x) d x
\end{aligned}
$$

then area difference at the moment:

$$
\begin{aligned}
\Delta S=S_{\tau 2}-S_{\tau 1}= & \int_{x_{l \tau 2}}^{x_{r \tau 2}} f_{\tau 2}(x) d x+\int_{x_{l \tau 2}}^{x_{r \tau 2}} \varphi_{\tau 2}(x) d x \\
& \quad-\int_{x_{l \tau 1}}^{x_{r \tau 1}} f_{\tau 1}(x) d x-\int_{x_{l \tau 1}}^{x_{r \tau 1}} \varphi_{\tau 1}(x) d x
\end{aligned}
$$

length of fire spreading when $y>0$ at time $\tau$

$$
L^{+}=\int_{x_{l \tau}}^{x_{r \tau}} \sqrt{1+\left(f_{\tau}^{\prime}\right)^{2}} d x
$$

In the same way length of fire spreading when $y<0$

$$
L^{-}=\int_{x_{l \tau}}^{x_{r \tau}} \sqrt{1+\left(\varphi_{\tau}^{\prime}\right)^{2}} d x
$$

then total length of fire spreading:

$$
L=L^{+}+L^{-}=\int_{x_{l \tau}}^{x_{r \tau}} \sqrt{1+\left(f_{\tau}^{\prime}\right)^{2}} d x+\int_{x_{l \tau}}^{x_{r \tau}} \sqrt{1+\left(\varphi_{\tau}^{\prime}\right)^{2}} d x
$$

\section{Simulation Experiments}

\subsection{Experiment area}

The experimental region is the city of Guangzhou, as an international flower city, which has complicated topography and plenty of lighting and heating resource. The forestland area in Guangdong has increase greatly after ten years' forestation and large-scale action of putting bare mountain to lush forest. There is a large proportion of middle age and young forest in Guangzhou; the tree population structure is haploid. In additional to dispersive natural village in suburb and frequently human being activities. so forest fire can occurs easily. 


\subsection{Data processing}

Data processing flows is shown in Figure 4.

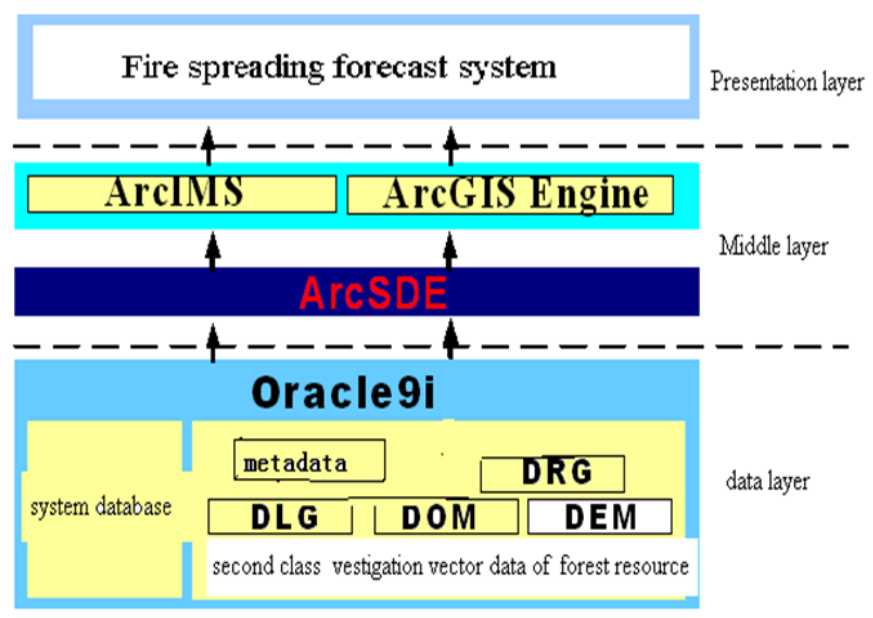

Figure 4. Data processing flow

(1) The original data. Includes large scale topographic map, ground control point file, fuel type distribution maps, and thematic maps. While making the combustible distribution diagram, different "layers" are made by GIS map information system according to altitude, slope, soil type, annual rainfall. Which make each layer link with a certain site vegetation. Through the combination, superposition, composite of different graph, it implement the splicing of the map, and the production of various complex thematic maps for fire spreading research.

(2) Metadata building. Includes the Gauss - Krueger projection zone, zoning, central meridian and standard parallel, and etc. Each subcompartment cards in forest base resources database stand for a record, in order to realize the connection of attribute database and graphics database, subcompartment database added a data item ID corresponding to the key words in graphic library, loading space database engine (ArcSDE). It also realizes the management of 3D data.

(3) Data acquisition. By scanning digitizing the topographic map in GIS software. It establish digital elevation model of various forest farm, get orthographic images and region model data and attribute data, such as road, lakes, and so on. it create the DEM elevation data with reference to elevation data, which including elevation point, contour, gully line (water system network), rivers, mountain ridges, Generating DEM data mainly adopts the ArcGIS software, and using the elevation data to create TIN, TIN can be converted to DEM.

(4) Registration. In order to found forest fire, it mainly rely on the remote sensing image, the registration between remote sensing image of fire dot and DEM image, which is the base to product three-dimensional relief image map. Due to the characteristics of registration completely by using valley lines to generate the DEM elevation data, we can use the valley lines to conduct geometric correction of remote sensing data, thereby ensuring the correctness of registration between remote sensing image and DEM image. Registration after the use of data base of forest resources can be inflammation of topography, natural object types

5) Data conversion. According to the terrain data, text data by means of simulation modeling 
software, after the completion of conversion process, and the successful implementation between all kinds of heterogeneous systems, will measure the data through GIS converted to create fire virtual environment required for the format of the data, such as digital elevation model data DEM, digital culture data DFD, texture data TIFF or JPG format.

\subsection{Multi-Agent decision-making and its implementation}

The spreading of fire is a kind of spatio-temporal phenomenon, more precisely expression, the integration of space process model and spatial data model .so the coupling of GIS and multi-agent is necessary. Common integration method (MAS model and GIS) fall into two categories: coupling And embedded method, two forms of coupling are loosely coupled and tightly coupled. This paper adopts embedding the necessary GIS function in MAS simulation system. According to the system characteristics and the functional requirements, the whole information system is illustrated in Figure 5.

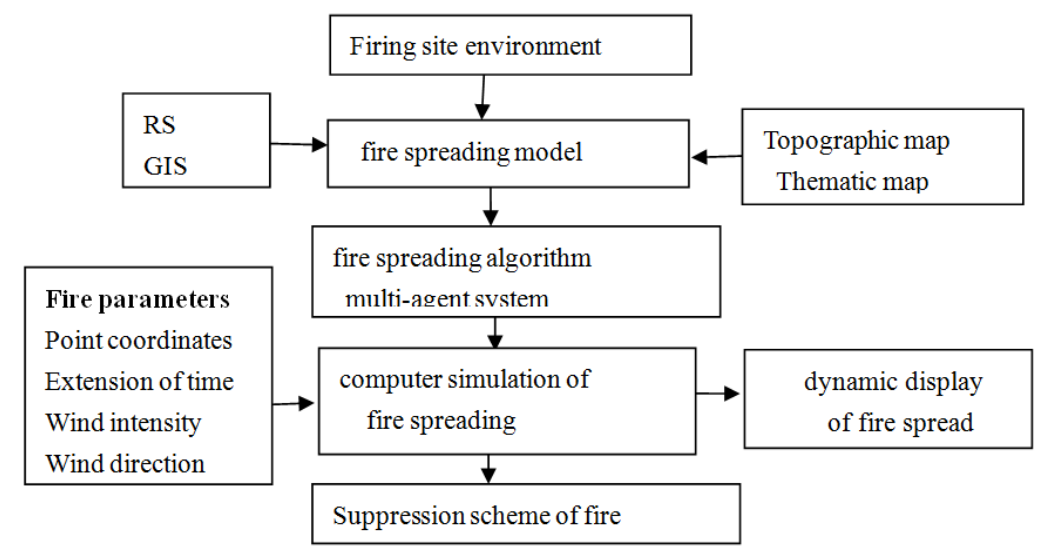

Figure 5. The flow chart of computer simulation for forest fire

Based on the simulation platform Repast and ArcGIS Engine, simulation system framework was built. Repast is a multi-agent modeling software, which can be used to simulate the natural and social phenomenon. With its own model libraries, users can simulate and model multi-agent systems by changing various conditions set. It is particularly suitable for multiple development of complex system. Modelers can put many orders to multiple agents at the same time; it also provides some built-in adaptation functions, to support internal system dynamic model and the discrete event parallelism Operation.

The model consists of a series of fire spreading related factors

$\mathrm{F}=\left(\mathrm{f}_{1,1}, \ldots \mathrm{f}_{\mathrm{i}, \mathrm{j}} \ldots \mathrm{f}_{\mathrm{n}, \mathrm{n}}\right)$

where $F$ represents the collection of all factors, $i$ and $j$ represent line and row numbers of cells, each cell is a discrete part of running environment and includes environment information $S_{i, j}$ which is expressed as below:

$S_{i, j}=\left(A_{i, j, 1}, A_{i, j, 2}, \ldots A_{i, j, n,}, R_{1}, R_{2}, B\right)$,

where $a_{i, j, 1}, a_{i, j, 2}, \ldots a_{i, j, n}$ represent land price, soil, terrain, traffic and other factors, and $n$ is the number of factors. $\mathrm{R}_{1}$ represents geographically bounded border in fire areas and the range of possible position of fire, which also a constraint of its movement. $R_{2}$ represents the position of road and river around the fire area, B represents the position of building and block around the fire area. 
Fire spreading agent $\mathrm{S}$-Agent $=\left\{\mathrm{Id}, \mathrm{P}_{0}, \mathrm{P}_{\mathrm{r}}, \mathrm{V}, \mathrm{P}\right.$, Rule, Query,Next $\}$

Where Id represents a unique identifier of the Agent ; $\mathrm{P}_{\mathrm{O}}$ represents starting position of fire spreading agent , $\mathrm{P}_{\mathrm{r}}$ represents the spreading location; $\mathrm{V}$ represents spreading speed of fire; $\mathrm{P}$ represents perceive state set; Rule represents the specific decision rule of fire spreading,

Query represents the agent perceive surrounding environment;

Next is determining the burning range at present based on the latest perceived state, combined with the rules corresponding to the burning environment.

According to the characteristics of forest fire behavior, temperature, humidity, wind speed, integrated wind and other meteorological factors, slope, aspect, slope position and geographic factors, as well as the fuel type, a simulation model is established, the formation of the real environment of forest fire, including the fire occurrence, development and spreading. The $3 \mathrm{~d}$ flight simulation is also established in this system; in any perspective users can feel the scene of the true fire environment. Simulation results as shown in Figure 6 and Figure 7.

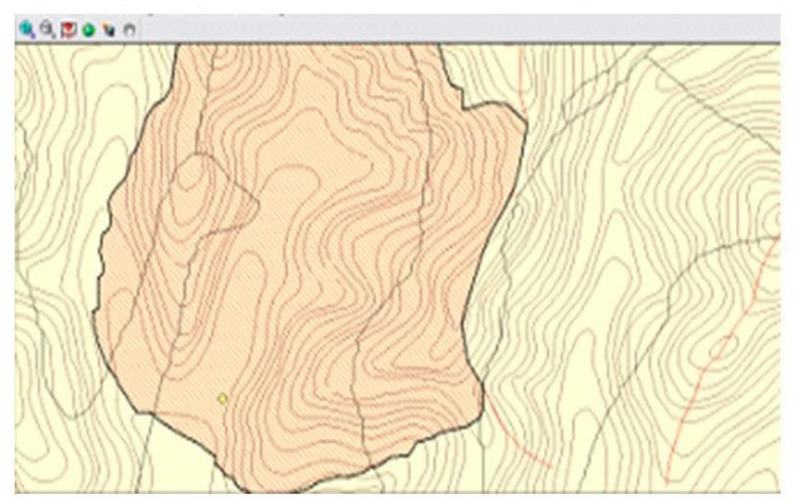

\section{Figure 6. Computer Simulation of Forest Fire}

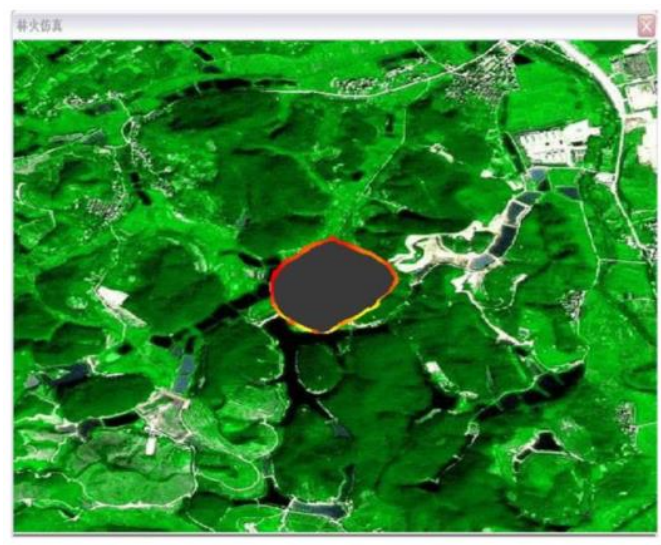

Figure 7. Visual Representation of Fire spreading

\section{Conclusions}

As far as firing prevention is concerned, it involved in many social factors. Firing prevention is a complicated systematic project needs long-time and difficult work, which is restricted by some factors such as society, environment and economy. With the increasingly 
responsibility of ecology environment construction, the requirements to fire prevention work is getting higher and higher.

In view of the present question, according to subjective initiative, learning ability and interaction characteristics of Multi-Agent Systems participating in forest fire spreading decision, an interactive model is put forward from integrating MAS and dynamic information feedback mechanism, and corresponding simulation experiment of fire suppression based on the model is also implemented. The system can monitor fire point in real time and extract forest fire in initial information through meteorological satellite ground received station that has been built.

\section{Acknowledgements}

This work is supported by the National Natural Science Foundation of China (Grant No.61273157).

\section{References}

[1] A. Ligtenberg, A. J. M. Beulens, D. L. Kettenis, A. K. Bregt and M. Wachowicz, "Simulating knowledge sharing in spatial planning:an agent-based approach", Environment and Planning B: Planning and Design, vol. 36, no. 4, (2009), pp. 644-663.

[2] L. Wang and F. Xiao, "A new approach to consensus problems indiscrete time multi-agent systems with time-delays", Science in China Series F: Information Sciences, vol. 50, no. 4, (2007), pp. 625-635.

[3] L. Fang and P. J. Antsaklis, "Asynchronous consensus protocols using nonlinear paracontractions theory", IEEE Transaction on Automatic Control, vol. 53, no. 10, (2008), pp. 2351-2355.

[4] Y. -p. Tian and C. -1. Liu, "Consensus of multi-agent systems with diverse input and communication delays", IEEE Transactionon Automatic Control, vol. 53, no. 9, (2008), pp. 2122-2128.

[5] X. F. Fan, X. W. Jiang, W. H. Huang, et al., "Research of multi-agent system based satellite fault diagnosis technology", Journal of Harbin Institute of Technology, vol. 9, no. 3, (2002), pp. 239-244.

[6] S. F. Yuan, X. S. Lai, X. Zhao, et al., "Distributed structural health monitoring system based on smart wireless sensor and multi-agent technology", Smart Materials and Structures, vol. 15, no. 1, (2006), pp. 1-18.

[7] M. Batty, "Agent-based Technologies and GIS: Simulating Crowding, Panic and Disaster Management", in Frontiers of Geographic Information Technology, Berlin: Springer-Verlay, (2006).

[8] F. Xiao and L. Wang, "Consensus protocols for discrete-timemulti-agent systems with time-varying delays", Automatica, vol. 44, no. 10, (2008), pp. 2577-2582.

[9] Y. -g. Sun and L. Wang, "Consensus of multiagent systems indirected networks with nonuniform time-varying delays”, IEEE Transaction on Automatic Control, vol. 54, no. 7, (2009), pp. 1607-1613.

[10] X. Fan, B. Sun, S. Sun, M. McNeese, J. Yen, R. Jones, T. Hanratty and L. Allender, "RPD-Enabled Agents Teaming with Humans for Multi-Context Decision Making", Proceedings of the Fifth International Joint Conference on Autonomous Agents and Multi-Agent Systems, Japan: AAMAS, (2006), pp. 34-41.

[11] R. G. Campbell, "Diagnostic reasoning agents development for HUMS Systems", Proc. of the 57th AHS International Annual Forum, Washington, USA, (2001), pp. 1-5.

[12] E. Albert, "Verified models of multi-agent systems for vehicles health management", Proc. of SPIE of Smart Structures and Materials Modeling Signal Processing and Control, (2005), pp. 5757. 